\title{
Actualización de la huella de carbono de la Universidad San Francisco de Quito USFQ para el año 2015
}

\author{
Felipe Salazar ${ }^{1}$, Melanie Valencia ${ }^{2}$, Alexandra Velasco ${ }^{2,3}$ y Valeria Ochoa-Herrera ${ }^{1,2}$ \\ 'Universidad San Francisco de Quito, Colegio de Ciencias e Ingeniería - El Politécnico, Diego de \\ Robles y Vía Interoceánica, Quito, Ecuador \\ ¿Universidad San Francisco de Quito, Oficina de Innovación y Sustentabilidad (OIS-USFQ), Diego de \\ Robles y Vía Interoceánica, Quito, Ecuador \\ ¿Universidad San Francisco de Quito, Colegio de Administración y Economía CADE, Diego de Robles \\ y Vía Interoceánica, Quito, Ecuador \\ *Corresponding author/ Autor para correspondencia, e-mail: vochoa@usfa.edu.ec
}

\section{Update of the carbon footprint of Universidad San Francisco de Quito - USFQ for the year 2015}

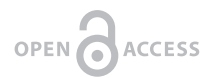

Editado por /

Edited by:

Diego F. Cisneros -

Heredia

Recibido /

Received:

2016/05/24

Aceptado /

Accepted:

2017/03/237

Publicado en línea / Published online: 2019/04/18

\section{Resumen}

La Universidad San Francisco de Quito USFQ en los últimos años se ha convertido en una institución comprometida con el desarrollo sostenible por lo que se realizó un nuevo estudio para evaluar las emisiones de carbono correspondientes al año 2015. La USFQ está seriamente comprometida en optimizar el manejo y consumo de agua, energía, combustible y desechos con el objetivo de convertirse en un campus inteligente. En este estudio la actualización de la huella de carbono correspondiente al año 2015 fue calculada y comparada con los datos obtenidos de la línea base correspondiente al año 2012. La huella de carbono fue calculada en base a una estimación de las emisiones másicas de $\mathrm{CO}_{2}$ generadas por distintos rubros correspondientes a actividades propias de la comunidad universitaria estudiantes, facultad y personal administrativo. Para este estudio, se consideraron las emisiones derivadas del consumo energético, la generación y tratamiento de aguas residuales, generación y tratamiento de residuos sólidos urbanos, consumo de combustibles, y transporte (terrestre y aéreo) de estudiantes, profesores y personal administrativo. Los datos fueron obtenidos de los registros de consumo de agua, electricidad, y combustibles. Adicionalmente, se realizó una encuesta electrónica para generar información primaria sobre el transporte de toda la comunidad USFQ. Diferentes metodologías de cálculo fueron aplicadas para determinar las emisiones de $\mathrm{CO}_{2}$ dependiendo del tipo de rubro analizado; cálculos basados en el método del factor de emisión para el consumo de combustible y energía, tratamiento biológico del agua residual y tratamiento de residuos urbanos. Los resultados finales indican que, en el 2015, cada miembro estudiantil contribuyó con una generación de 0.68 t CO 2 al año, y cada miembro docente y administrativo contribuyó con una generación de 0.84 t $\mathrm{CO}_{2}$ al año. Comparando con la línea base 2012, se encontró una reducción en la emisión 
por estudiante por un factor de 1.18. En total, la USFQ emite hacia la atmósfera 6225.4 t $\mathrm{CO}_{2}$ anuales, siendo la mayor contribución el transporte terrestre (incluyendo toda la comunidad) con un aporte del 68.0\%, seguido por la generación de energía 17.1\%, transporte aéreo $8.6 \%$, tratamiento de residuos sólidos urbanos 4.2\%, combustión de combustibles in-situ $1.5 \%$, y tratamiento de agua residual $0.6 \%$.

Palabras clave: Huella de carbono, emisiones de $\mathrm{CO}_{2^{\prime}}$ USFQ, $\mathrm{CO}_{2} \mathrm{CH}_{4^{\prime}}$ sostenibilidad

\section{Abstract}

Universidad San Francisco de Quito (USFQ), in the last years, had become an institution committed to sustainable development. In order to fulfill this commitment, a new study was conducted to evaluate our own carbon emissions corresponding to the year 2015. USFQ is determined to become a smart campus by optimizing water, energy, fuel, and waste management. In this study, USFQ's carbon footprint was calculated for the year 2015 and compared to data obtained from the baseline year of 2012. The C-foot-print was calculated based on an estimate of the $\mathrm{CO} 2$ mass emissions generated by the different operational areas within the university and all of its users (students, faculty, and other administrative bodies). The CO2 emissions were calculated from the following activities: energy consumption, wastewater generation, solid waste generation, fuel consumption, and the users' mobility (land and air modes of transportation). The data was collected from the different consumption records including those concerning water, energy, and fuel consumption. In addition, an electronic survey was conducted to generate primary information about the transportation of USFQ community. Different methodologies and calculations were applied depending on the source analyzed such as factor emission based calculations for fuel and power consumption, biological treatment of wastewater, and treatment of solid waste. The final results indicate that in 2015 each student contributed to 0.68 t CO2, while faculty and administrative bodies contributed to 0.84 t CO2. Comparing these results to baseline the year 2012, we found a reduction by a factor of 1.18 of the emissions contributed by each student. Overall, Universidad San Francisco de Quito USFQ has emitted to the atmosphere a total of $6225.4 \mathrm{t} \mathrm{CO} 2$, from which the largest contribution comes from the land mobility (including all users), representing a $68 \%$ of this total value; followed by $17.1 \%$ from electric consumption, $8.6 \%$ from air transportation, $4.2 \%$ from solid waste treatment, $1.5 \%$ from fuel combustion in-situ and $0.6 \%$ from wastewater treatment.

Key words: carbon footprint, $\mathrm{CO}_{2}$ emissions, USFQ, $\mathrm{CO}_{2^{\prime}} \mathrm{CH}_{4^{\prime}}$ sostenibilidad

\section{INTRODUCCIÓN}

El dióxido de carbono $\left(\mathrm{CO}_{2}\right)$ es el principal gas de efecto invernadero (GEI) antropogénico que contribuye al incremento del nivel total de concentración de GEl en la atmósfera, y por lo tanto al cambio climático [1]. Este gas se encuentra de manera natural en la atmósfera debido al ciclo del carbón [2] y de acuerdo al IPCC (Intergovernmental Panel on Climate Change), las fuentes principales de $\mathrm{CO}_{2}$ antropogénico son la generación de electricidad, transporte terrestre, aéreo, y marítimo, actividades industriales, actividades comerciales y residencialeses, y otras combustiones no asociadas a combustibles fósiles 
[1]. En las últimas décadas, la concentración de $\mathrm{CO}_{2}$ en la atmósfera ha aumentado y en la actualidad sobrepasa los 400 ppm, el 97\% de los científicos expertos en cambio climático concuerdan que existe suficiente evidencia científica de que este aumento se debe a las actividades antropogénicas [3]. Siendo las emisiones de $\mathrm{CO}_{2}$, un factor de gran influencia sobre el cambio climático, es imprescindible que las instituciones de educación superior (IES) calculen su huella de carbono para conocer la magnitud en la cual estas entidades aportan al cambio climático y contribuir al desarrollo sustentable [4]. En los últimos años las IES han reconocido la importancia de incluir conceptos de sustentabilidad y protección del medio ambiente en la educación de sus estudiantes debido a su posición única de promover el cambio y formar a los líderes de las nuevas generaciones [5].

En el año 2013 la Universidad San Francisco de Quito USFQ se unió al Piloto Internacional de Association for the Advancement of Sustainability in Higher Education (AASHE) y empleó el programa Sustainability Tracking, Assesment \& Rating System (STARS) para evaluar su desempeño en sustentabilidad y calcular su huella de carbono [6]. STARS ha sido reconocida internacionalmente como el programa más completo para evaluar la sustentabilidad en instituciones de educación superior [7]. En el Primer Reporte de Sustentabilidad de la Universidad San Francisco de Quito se realizó el cálculo de las emisiones másicas totales de $\mathrm{CO}_{2}$ asociadas a las actividades de la población estudiantil, docente y administrativa correspondiente al año 2012. La huella de carbono fue calculada analizando diferentes rubros por separado para conocer el aporte de cada uno de ellos y poder en un futuro cercano establecer las áreas prioritarias para reducir el impacto al medio ambiente y caminar hacia convertirse en un campus inteligente. Las emisiones totales de $\mathrm{CO}_{2}$ de la USFQ en al año 2012 fueron $4750.7 \mathrm{tCO}_{2}$, equivalentes a $0.8 \mathrm{t} \mathrm{CO}_{2}$ por estudiante.

El objetivo principal de este estudio fue actualizar la huella de carbono de la Universidad San Francisco de Quito, USFQ, para el año 2015 y compararla con los datos de línea base obtenidos en el año 2012. En este estudio se presentan los cálculos de las emisiones de $\mathrm{CO}_{2}$ correspondientes a la USFQ por los rubros de energía, consumo de combustibles, transporte terrestre y aéreo, tratamiento biológico de las aguas residuales, y tratamiento de residuos sólidos urbanos (RSU). La actualización de la huella de carbono permite evaluar el desempeño actual de la USFQ y llevar a cabo acciones a futuro con el objetivo de optimizar operaciones y desarrollar programas sustentables que permitan generar un ahorro energético, de agua y de los recursos en general y educar a la comunidad en temas de sustentabilidad. Adicionalmente, la USFQ es la primera universidad en Sudamérica en reportar para STARS y ser parte de la comunidad AASHE. De acuerdo a nuestro conocimiento, no existen datos publicados en la literatura sobre huellas de carbono de otras universidades en la región por lo que los datos obtenidos en este estudio son de gran importancia para contribuir al desarrollo sustentable en instituciones de educación superior en Latinoamérica.

\section{MATERIALES Y MÉTODOS}

El cálculo de la huella de carbono de la USFQ se realizó tomando en consideración tres diferentes alcances y fue restringido al campus Cumbayá [1]. El alcance 1 abordó las emisiones directas de GEl que se dan dentro de la USFQ tales como el consumo de diésel, 
gas licuado de petróleo (GLP), gasolina (nafta) y $\mathrm{CH}_{4}$ emitido de desechos orgánicos. El alcance 2 contempló las emisiones indirectas de GEl asociadas a la energía comprada y consumida por la USFQ. El alcance 3 correspondió a otras emisiones indirectas que no están asociadas con el consumo energético que incluyeron la cantidad de $\mathrm{CO}_{2}$ emitida del tratamiento de residuos sólidos urbanos (RSU), transporte terrestre y viajes aéreos de los estudiantes, facultad y personal administrativo; y las emisiones asociadas al consumo, generación y tratamiento de las aguas residuales (AR). Los datos empleados corresponden al segundo semestre 2014-2015 (enero a mayo), verano 2015 (junio y julio), y primer semestre 2015-2016 (agosto a diciembre). Los datos para los rubros de transporte terrestre y aéreo fueron obtenidos a partir de una encuesta electrónica realizada a toda la población estudiantil y facultad en noviembre del 2015.

\section{Generación de emisiones de $\mathrm{CO}_{2}$ a partir del consumo de energía}

Las emisiones de $\mathrm{CO}_{2}$ por compra y uso de electricidad dentro de la universidad fueron calculadas utilizando la metodología desarrollada por Parra que se encuentra descrita en el Primer Reporte de Sustentabilidad de la USFQ [6]. Los datos fueron proporcionados por el proveedor público local "Empresa Eléctrica Quito SA". El factor de emisión fue calculado siguiendo la metodología propuesta por Parra [8] y fue $342.3 \mathrm{~g} \mathrm{CO}_{2} \mathrm{kWh}^{-1}$ para el año 2014.

\section{Generación de emisiones de $\mathrm{CO}_{2}$ a partir del consumo de combustibles}

Los datos del consumo de combustibles fueron obtenidos del registro universitario interno de pagos. El cálculo de emisión de $\mathrm{CO}_{2}$ por el consumo de combustibles se realizó teniendo en consideración los tres tipos de combustibles que la universidad compra por separado que son gasolina (nafta), diésel y GLP de acuerdo a lo establecido por Parra en el reporte de sustentabilidad de la USFQ [6].

\section{Generación de emisiones de $\mathrm{CO}_{2}$ a partir del tratamiento del AR}

Los datos de consumo de agua fueron proporcionados por la Empresa Pública Metropolitana de Agua Potable y Saneamiento (EPMAPS). La metodología para el cálculo de las emisiones de $\mathrm{CO}_{2}$ por este rubro se basa en la reacción de oxidación aeróbica de la materia orgánica descrita por Eddy, et al., [9] y desarrollada por OchoaHerrera en el Primer Reporte de Sustentabilidad de la USFQ [6]. El valor de demanda química de oxígeno (DQO) $\left(0.9 \mathrm{~g} \mathrm{~L}^{-1}\right)$ del AR doméstica generada en Quito empleado fue determinado por Benítez [10] y se asumió un 90\% biodegradabilidad [9].

\section{Generación de emisiones de $\mathrm{CO}_{2}$ a partir del tratamiento de $\mathrm{RSU}$}

La generación de emisiones de $\mathrm{CO}_{2}$ por el tratamiento de residuos fue calculada a partir de la metodología desarrollada por Peñafiel descrita en el Primer Reporte de Sustentabilidad de la USFQ [6]. Los desechos generados fueron tomados y caracterizados un día al azar durante el periodo de enero-mayo 2015 (Segundo semestre 2014-2015). El año estudiantil empleado fue de 227 días de clase divididos en tres periodos estudiantiles. Los siguientes períodos fueron corregidos en base al número de días, profesores y estudiantes. En el periodo junio-julio existieron 44 días de clase, 3172 estudiantes y 
409 profesores/personal administrativo, mientras que en el periodo agosto-diciembre existieron 92 días de clase, 8025 estudiantes, y 913 profesores/personal administrativo. Una vez obtenida la cantidad total de RSU generados sin contar el porcentaje reciclado, la cantidad teórica de biogás que se generaría durante la estabilización de los RSU en el relleno sanitario fue calculada a partir de la metodología propuesta por Tchobanoglous, et al., [11] y desarrollada por Peñafiel [6]. La equivalencia de $\mathrm{CH}_{4}$ en $\mathrm{CO}_{2}$ eq se obtuvo a partir del factor potencial de cambio climático del metano (21), establecido en el informe del IPCC [12].

\section{Generación de emisiones de $\mathrm{CO}_{2}$ a partir de transporte}

Las emisiones de $\mathrm{CO}_{2}$ a partir del transporte terrestrey aéreo de los miembros de la USFQ fueron calculadas en base a la metodología propuesta por Cazorla y colaboradores [6, $13,14]$. En el caso del transporte terrestre la información sobre el tipo de transporte fue generada a partir de una encuesta electrónica realizada en noviembre del 2015. Una vez obtenida una muestra referencial del 70\% de la población estudiantil (5618 encuestas), se prosiguió a filtrar las encuestas correctamente respondidas, obteniendo un número final de 1500 encuestas. La emisión másica promedio de $\mathrm{CO}_{2}$ por persona por semana fue extrapolada para 8025 personas, que fue la población estudiantil total de la USFQ en el primer semestre 2015-2016. El cálculo para las emisiones asociadas al transporte de la facultad docente de la universidad fue calculado de la misma manera y los datos se obtuvieron a partir de 120 encuestas filtradas, correspondientes a una muestra representativa del $13.17 \%$ de la población docente en la USFQ. Las emisiones de $\mathrm{CO}_{2}$ por el transporte aéreo de los viajes nacionales e internacionales realizados por los estudiantes regulares, estudiantes de intercambio (extranjeros y nacionales), facultad y personal administrativo fueron calculadas con la metodología desarrollada por Cazorla y colaboradores $[6,13,14]$ en base a las recomendaciones realizadas por la Organización Internacional de Aviación Civil (ICAO) [15]. En el caso de los viajes de los docentes y personal administrativo se asumió viajes completos (ida y vuelta) para los traslados nacionales, internacionales y a las estaciones científicas. Con respecto a los estudiantes locales de intercambio, se asumió viajes completos solamente para los estudiantes que viajaron al exterior y regresaron dentro del mismo año (estudiantes pertenecientes a la USFQ). Mientras que para los estudiantes internacionales que ingresaron a la USFQ de intercambio solo se asumió el vuelo de llegada. Cabe indicar que, con respecto a la facultad, no se incluyeron los viajes terrestres que son aquellos que más contribuyeron a las emisiones del 2012 ya que esta información no pudo ser obtenida para este estudio.

\section{RESULTADOS Y DISCUSIÓN}

La huella de carbono de la USFQ fue actualizada para el año 2015 de acuerdo a la metodología descrita en la sección de Materiales y Métodos. En la Tabla 1 se presenta un resumen de las emisiones totales de $\mathrm{CO}_{2}$ de la USFQ divididas por alcance con un total de emisiones de 6225.4 t CO 2 en el 2015, siendo las emisiones por transporte terrestre y aéreo equivalentes al $76.6 \%$ de la emisión total. 
La energía eléctrica total consumida proveniente de la red pública fue 3106.3 MWh para el año 2015 en la USFQ campus Cumbayá, con un promedio de consumo mensual de 258.9 MWh. En la Fig. 1 se presenta las emisiones de $\mathrm{CO}_{2}$ mensuales $\left(\mathrm{CO}_{2}\right)$ en la USFQ asociadas al rubro energético. Evidentemente, en los períodos estudiantiles ordinarios entre enero y junio, y septiembre y diciembre existe un mayor aporte de emisiones. En los meses de julio y agosto (verano) se observa un menor consumo energético, y por ende un menor aporte de emisión. Las emisiones de $\mathrm{CO}_{2}$ por consumo energético contribuyeron con un $17.1 \%$ a las emisiones totales de $\mathrm{CO}_{2}$ (Tabla 1). Las emisiones de $\mathrm{CO}_{2}$ por este rubro fueron 1.2 veces mayor que aquellas reportadas en el 2012. Sin embargo, este factor no es tan elevado tomando en cuenta que la población estudiantil incrementó de 5925 a 8025 estudiantes entre estos dos periodos.

TABLA 1: Emisiones de $\mathrm{CO}_{2}$ por alcances 1,2 y 3 de la Universidad San Francisco de Quito USFQ correspondientes al año 2015

\begin{tabular}{|c|c|c|}
\hline Alcance & $\mathrm{tCO}_{2}$ & Porcentaje (\%) \\
\hline Total del Alcance 1 & 93.2 & 1.5 \\
\hline Diesel & 24.5 & 0.4 \\
\hline GLP & 43.9 & 0.7 \\
\hline Combustible (Gasolina) & 24.8 & 0.4 \\
\hline Total del Alcance 2 & 1063.3 & 17.1 \\
\hline Electricidad comprada & 1063.3 & 17.1 \\
\hline Total del Alcance 3 & 5068.9 & 81.4 \\
\hline $\mathrm{CO}_{2}$ emitido de desechos & 28.6 & 0.5 \\
\hline $\mathrm{CH}_{4}$ emitido de desechos* & 235.0 & 3.8 \\
\hline Transporte terrestre de estudiantes (auto + bus) & 3695.0 & 59.4 \\
\hline Transporte facultad y personal de servicio (auto + bus) & 537.8 & 8.6 \\
\hline Viajes estudiantes de intercambio USFQ & 246.6 & 4.0 \\
\hline Viajes estudiantes de intercambio del extranjero & 143.6 & 2.3 \\
\hline Viajes de negocio a Tiputini/ Galápagos & 69.2 & 1.1 \\
\hline Viajes de negocio resto del Ecuador & 2.8 & 0.0 \\
\hline Viajes de negocio al extranjero & 75.2 & 1.2 \\
\hline Tratamiento de agua residual & 35.2 & 0.6 \\
\hline Total & 6225.4 & 100 \\
\hline
\end{tabular}


FIGURA 1: Emisiones de $\mathrm{CO}_{2}$ mensuales ( $\left.\mathrm{t} \mathrm{CO}_{2}\right)$ en la USFQ campus Cumbayá asociadas al rubro energético correspondientes al año 2015

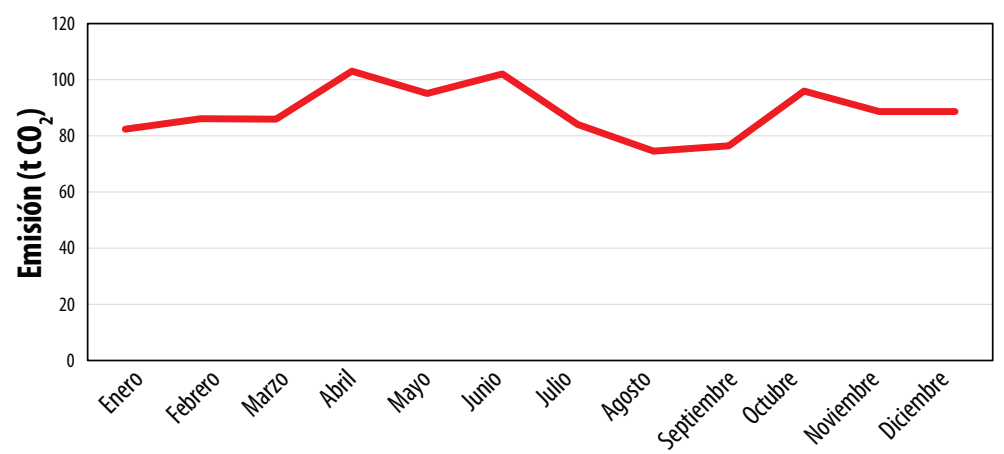

La cantidad total de agua potable consumida en el año 2015 fue 28268.4 m³$^{3}$, con un consumo promedio mensual de $2355.7 \mathrm{~m}^{3}$. En la Fig. 2 se presenta las emisiones teóricas mensuales de $\mathrm{CO}_{2}$ correspondientes al año 2015 asociadas al tratamiento de aguas residuales domésticas. En la Fig. 2 se puede observar un suceso ambiguo al obtenido para las emisiones de $\mathrm{CO}_{2}$ por consumo energético en los meses de verano (julio y agosto) que es el período en el que la universidad se encuentra en receso académico. En dicho período se registra un incremento significativo en las emisiones de $\mathrm{CO}_{2}$, las cuales están directamente relacionadas con un incremento en el consumo de agua. De acuerdo al Departamento de Planta Física de la USFQ, el consumo de agua en el verano se debió a una fisura dentro de la laguna artificial de la universidad que generó una pérdida sustancial de agua y la laguna fue llenada diariamente. A partir del mes de septiembre se observa una emisión estable de $\mathrm{CO}_{2}$ debido a que en este mes se realizaron unas adecuaciones para tratar de cerrar las fisurar encontradas. Cabe indicar que las emisiones teóricas de $\mathrm{CO}_{2}$ provenientes del tratamiento biológico de AR no contribuyen de forma importante a la emisión total correspondiente al año 2015. Sin embargo, es importante resaltar que este valor fue 2.15 veces mayor que aquel obtenido en el 2012 [6].

FIGURA 2: Emisiones de $\mathrm{CO}_{2}$ mensuales ( $\mathrm{CO}_{2}$ ) en la USFQ campus Cumbayá asociadas al rubro tratamiento de AR correspondientes al año 2015

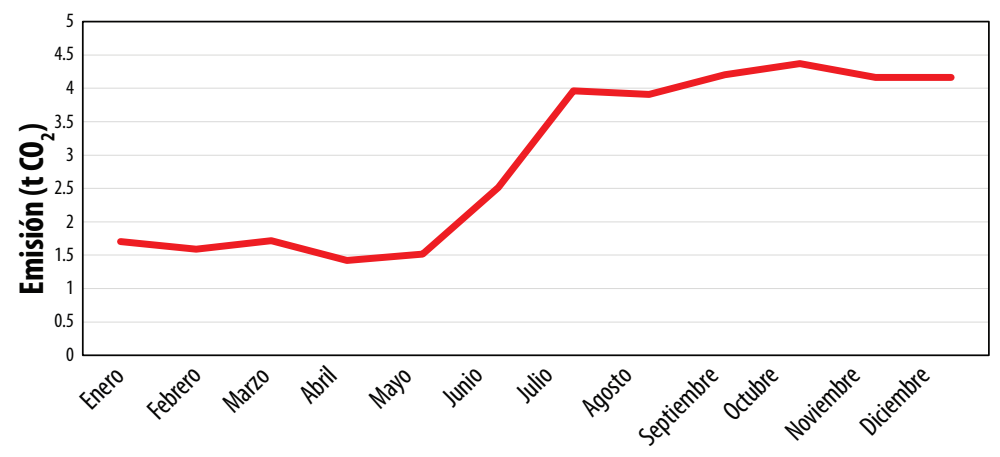


Las emisiones correspondientes al consumo total de combustibles utilizados para generadores eléctricos y restaurantes en la USFQ representan apenas un $1.5 \%$ de las emisiones totales de $\mathrm{CO}_{2}$ (Tabla 1). Las emisiones debido a la gasolina (nafta) utilizada se mantuvo estable con respecto a la línea base del 2012, mientras que las emisiones correspondientes al consumo de diésel y GLP disminuyeron por factores de 4.32 y 2.34 , respectivamente en el 2015 [6]. Esta reducción en las emisiones de $\mathrm{CO}_{2}$ está probablemente asociada a la compra de combustibles ya que de acuerdo a la información proporcionada por el personal de mantenimiento en los meses de enero y julio no se realizó una compra de diésel y LPG, lo cual sugiere que en el 2014 hubo un exceso de combustible comprado y este fue utilizado en los primeros meses del año 2015.

En el caso de los desechos sólidos, la recolección total fue de 139.6 t en el año 2015 (Tabla 2) y estos fueron caracterizados como $47 \%$ residuos orgánicos, $18 \%$ de residuos de papel, $15 \%$ de residuos de jardín, 10\% de plástico mezclado, 4\% vidrio, 3\% otros, y $1 \%$ cartón, madera y metal y latas. Los RSU generados en el 2015 fueron 1.3 veces menores a aquellos reportados en el año base 2012. Varias razones pueden contribuir a esta reducción en la generación de RSU. En primer lugar, en el último año se recicló mayor cantidad de residuos lo cual podría sugerir que la universidad está concientizando a su comunidad en la generación de RSU. Adicionalmente, la USFQ implementó clases en el Paseo San Francisco, por lo que probablemente parte de los desechos pudieron ser colocados en las instalaciones del Paseo y en este estudio no se incluyó las emisiones generadas en esta localidad. Las emisiones teóricas de $\mathrm{CO}_{2}$ directas equivalieron al $0.5 \%$ de las emisiones totales. Las emisiones de $\mathrm{CH}_{4}$ por la estabilización de los RSU aportaron con un $3.8 \%$ al total de todos los rubros analizados.

TABLA 2: Cantidad de RSU producidos en el 2015 (toneladas)

\begin{tabular}{|c|c|}
\hline Periodo Calendario 2015 & Toneladas (t) \\
\hline Enero-Mayo & 59.9 \\
\hline Junio-Julio & 12.8 \\
\hline Agosto-Diciembre & 66.9 \\
\hline Total & 139.6 \\
\hline
\end{tabular}

Las emisiones de $\mathrm{CO}_{2}$ generadas por el transporte de la comunidad universitaria contribuyeron significativamente a la huella de carbono de la USFQ tanto en el 2012 como en el 2015. En la Figura 3 se presenta el modo de transporte terrestre de la población estudiantil, profesores y personal administrativo para el año 2015. Cabe indicar que del $49.2 \%$ de estudiantes que se movilizaron en automóvil o motocicleta, el $75 \%$ viajaron solos y el $25 \%$ compartieron su auto con otros estudiantes. En términos de la frecuencia de viajes, el $18.3 \%$ de estudiantes realizaron más de 10 viajes semanales, el 64\% realizaron 10 viajes semanales, y el $17.7 \%$ realizan menos de 10 viajes a la semana. Con respecto a los profesores y personal administrativo, el 77.5\% utilizaron automóvil y motocicleta a diario para transportarse desde y hacia la universidad. De esta población, el 39\% compartieron su automóvil o motocicleta y el 61\% viajaron solos (Fig.3) 
FIGURA 3: Distribución de medio de transporte estudiantil (barras negras), profesores y personal administrativo (barras blancas) correspondiente al año 2015.

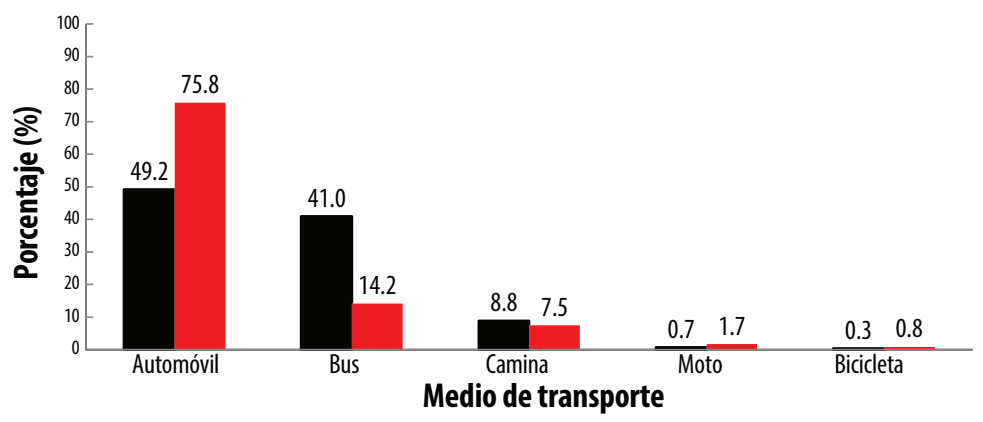

En la Tabla 3 se resume las toneladas de $\mathrm{CO}_{2}$ generadas por el transporte nacional e internacional de la comunidad USFQ en el 2015 con un total de 4770.1 t CO (Tabla $^{2}$ 1). El transporte terrestre de la comunidad universitaria contribuyó con un $68 \%$ de todos los rubros analizados, siendo esta la mayor fuente de emisión de $\mathrm{CO}_{2}$ en la USFQ

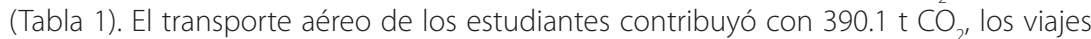
internacionales realizados por la facultad aportaron con un total de $75.2 \mathrm{tCO}_{2}$ y los viajes nacionales añadieron $2.8 \mathrm{t} \mathrm{CO}_{2}$. Finalmente, los viajes realizados a las extensiones de investigación de la USFQ en Galápagos y Tiputini, contribuyeron con una emisión de 69.2 t CO$_{2}$. Es importante recalcar que en el rubro transporte, el $88.7 \%$ corresponde al transporte terrestre y el $11.3 \%$ al transporte aéreo. Las emisiones asociadas al transporte de estudiantes en el año 2015 es 1.4 veces mayor a aquella obtenida en el 2012, mientras que las emisiones del transporte de docentes y personal administrativo es mayor por un factor de 1.7 veces [6]. Estos datos son coherentes con un incremento de la población estudiantil, de facultad y personal administrativo en el 2015.

TABLA 3: Toneladas de $\mathrm{CO}_{2}$ generadas por rubro de transporte de la comunidad USFQ correspondientes al año 2015

\begin{tabular}{|c|c|c|}
\hline Transporte & \multicolumn{2}{c|}{$\mathbf{t} \mathrm{CO}_{2}$} \\
\hline Carro & Estudiantes & Facultad/Personal \\
\hline Bus & 3460.6 & 530.8 \\
\hline Avión & 234.4 & 7.0 \\
\hline Subtotal & 452.3 & 85.0 \\
\hline Total & 4147.3 & 622.8 \\
\hline
\end{tabular}

En términos generales los resultados muestran las emisiones másicas totales de $\mathrm{CO}_{2}$ de la USFQ han aumentado desde el año 2012 hasta el año 2015. Uno de los factores principales que contribuyeron al aumento de las emisiones de $\mathrm{CO}_{2}$ fue la diferencia entre la cantidad de estudiantes, docentes y administrativos. En el año 2012 la USFQ contaba con 5925 estudiantes y con 450 profesores y personal; mientras que en el 2015, 
la USFQ contó con 8025 estudiantes y con 913 profesores y personal administrativo [6]. Probablemente, este incremento por un factor de 1.4 en el caso de estudiantes y 2.0 en el caso de docentes y personal administrativo ha contribuido en gran medida al incremento en los diferentes rubros analizados. Sin embargo, las emisiones de $\mathrm{CO}_{2}$ por unidad funcional han disminuido en el año 2015. En el Primer Reporte de Sustentabilidad de la USFQ se calculó que cada estudiante fue responsable por 0.8 t $\mathrm{CO}_{2}$ mientras que en el año 2015 cada estudiante y personal (docente y administrativo) es responsable por una emisión de 0.68 y 0.84 t $\mathrm{CO}_{2^{\prime}}$ respectivamente [6]. Esta diminución de 1.18 veces sugiere que el crecimiento en la cantidad de estudiantes en la USFQ es mucho mayor que el incremento en la emisión total por parte de los mismos.

TABLA 4: Emisiones de $\mathrm{CO}_{2}$ por estudiante y personal (docente y administrativo) correspondientes al año 2015

\begin{tabular}{|c|c|c|}
\hline Rubros & $\begin{array}{c}\mathrm{tCO}_{2} / \\
\text { estudiante }\end{array}$ & $\begin{array}{c}\mathrm{tCO}_{2} / \\
\text { personal }\end{array}$ \\
\hline Transporte terrestre & 0.460 & 0.589 \\
\hline $\begin{array}{l}\text { Transporte aéreo } \\
\text { Estudiantes (intercambio), Facultad (internacionales) }\end{array}$ & 0.049 & 0.082 \\
\hline Transporte aéreo (Galápagos y Tiputini) & 0.008 & 0.008 \\
\hline Transporte aéreo (nacionales) & - & 0.003 \\
\hline Consumo energético & 0.119 & 0.119 \\
\hline Generación de aguas residuales & 0.004 & 0.004 \\
\hline Consumo de combustible & 0.003 & 0.003 \\
\hline Consumo de diésel & 0.003 & 0.003 \\
\hline Consumo de GLP & 0.005 & 0.005 \\
\hline Generación de $\mathrm{CH}_{4}$ de RSU & 0.026 & 0.026 \\
\hline Generación de $\mathrm{CO}_{2}$ de $\mathrm{RSU}$ & 0.003 & 0.003 \\
\hline Total & 0.68 & 0.84 \\
\hline
\end{tabular}

Debido a que el rubro de transporte de estudiantes representa la mayor emisión de $\mathrm{CO}_{2^{\prime}}$ varios esfuerzos tales como la implementación y optimización del programa de auto compartido y/o la motivación de utilizar el transporte público están siendo realizados para reducir estas emisiones. Cabe recalcar que menos del 50\% de estudiantes comparten su auto con otros estudiantes en sus viajes semanales a la USFQ. De acuerdo a la literatura, las tres razones primordiales por las cuales los estudiantes usualmente prefieren viajar solos son a) falta de compañeros que estén dispuestos a compartir su auto, b) conflicto de horarios y c) diversas necesidades extracurriculares antes y después de clases, y aparentemente el principal incentivo que motiva a los estudiantes a participar en programas de auto compartido es el aporte económico para los servicios 
de estacionamiento [16]. Por lo tanto, la USFQ podría aplicar un servicio en el cual los estudiantes, facultad y administrativos que compartan su auto tengan estacionamiento asegurado. La utilización del programa auto compartido que es un plataforma comprada y adaptada por la universidad permite a la comunidad universitaria compartir el auto y de esta manera contribuir a reducir la huella de carbono de la USFQ y caminar hacia el objetivo de convertirse en un campus sustentable e inteligente.

En conclusión, los resultados muestran que en general, las emisiones totales de $\mathrm{CO}_{2}$ de la USFQ han aumentado desde el año 2012 hasta el año 2015 por un factor de 1.4 y 2.0 para estudiantes, facultad y administrativos, respectivamente. El incremento en el número de estudiantes, profesores y personal administrativo han contribuido indudablemente a que las emisiones de $\mathrm{CO}_{2}$ aumenten. El cálculo de la huella de carbono es un aporte fundamental al Reporte de Sustentabilidad de la USFQ ya que permite conocer las emisiones de $\mathrm{CO}_{2}$ hacia la atmósfera; y por consecuencia, la contribución al cambio climático de la universidad. Adicionalmente, permite establecer las áreas prioritarias en las que se deben enfocar los esfuerzos para reducir el impacto ambiental de las operaciones de la universidad. En vista que el transporte por medio terrestre es el rubro que más contribuye a las emisiones de $\mathrm{CO}_{2^{\prime}}$ la USFQ está trabajando en implementar programas que permitan optimizar la movilización de la comunidad universitaria. Adicionalmente, se están desarrollando programas de eficiencia energética, consumo responsable de agua, planes de manejo de residuos y reciclaje con el objetivo de convertirnos en un campus inteligente. El desarrollo sostenible dentro de la USFQ puede jugar un papel fundamental en el futuro de nuestros graduados y docentes. Todo esto contribuye a que la USFQ se esté estableciendo como un modelo a seguir en desarrollo sostenible en instituciones de educación superior en el país y en la región Andina.

\section{AGRADECIMIENTOS}

Expresamos nuestro profundo agradecimiento a todos los miembros de la comunidad universitaria de la USFQ, estudiantes, profesores y personal administrativo, que proporcionaron información valiosa y participaron activamente en este estudio. Un agradecimiento especial a Denisse Dalgo por su apoyo en la recolección de información, y a Ana Gisell Pazmiño por leer este manuscrito y revisarlo en repetidas ocasiones.

\section{CONTRIBUCIONES DE LOS AUTORES}

Valeria Ochoa-Herrera y Alexandra Velasco concibieron la investigación. Valeria OchoaHerrera administró el proyecto y lideró la adquisición de fondos y recursos. Felipe Salazar y Melanie Valencia realizaron la curación de datos para la investigación. Valeria Ochoa-Herrera estructuró y definió el enfoque del manuscrito. Felipe Salazar y Melanie Valencia redactaron el manuscrito. Valeria Ochoa-Herrera y Alexandra Velasco revisaron críticamente el contenido intelectual del manuscrito. Felipe Salazar realizó la producción de tablas y figuras. 


\section{REFERENCIAS}

[1] EPA, U. (2016). Inventory of US greenhouse gas emissions and sinks: 1990-2014.Recuperado el 19 de mayo de 2017 de: https://www.epa.gov/sites/production/files/2017-04/documents/us-ghg-inventory-2016-main-text.pdf (verified April 15, 2016).

[2] Watson, R. T., Rodhe, H., Oeschger, H., \& Siegenthaler, U. (1990). Greenhouse gases and aerosols. Climate change: the IPCC scientific assessment, 1, 17.

[3] Cook, J., Oreskes, N., Doran, P. T., Anderegg, W. R., Verheggen, B., Maibach, E. W., ... \& Nuccitelli, D. (2016). Consensus on consensus: a synthesis of consensus estimates on human-caused global warming. Environmental Research Letters, 11(4), 048002.

[4] Matthews, H. S., Hendrickson, C. T., \& Weber, C. L. (2008). The importance of carbon footprint estimation boundaries. 42(16): p. 5839-5842.

[5] Tilbury, D. (2011). Higher education for sustainability: a global overview of commitment and progress. Higher education in the world, 4, 1-28.

[6] Velasco, A., Ochoa-Herrera, V., Peñafiel, R., Cazorla, M. D. C., Parra, R., Naranjo, H., \& Valdés, A. (2014). Sustainability Report Universidad San Francisco de Quito: baseline year 2012. p. 1-97

[7] Sayed, A., Kamal, M., \& Asmuss, M. (2013). Benchmarking tools for assessing and tracking sustainability in higher educational institutions: identifying an effective tool for the University of Saskatchewan. International Journal of Sustainability in Higher Education, 14(4), 449-465.

[8] Parra, R. (2013). Factor de emisión de C02 debido a la generación de electricidad en el Ecuador durante el período 2001-2011. Avances, 5(1), C39-C42.

[9] Eddy, M. A., Burton, F. L., Tchobanoglous, G., \& Tsuchihashi, R. (2013). Wastewater engineering: treatment and resource recovery (p. 2048). McGraw-Hill Education: New York, NY, USA.

[10] Benítez Vallejo, M. B. (2015). Wastewater treatment for nutrient recovery with Ecuadorian native microalgae (Bachelorss thesis, Quito: USFQ, 2015).

[11] Tchobanoglous, G., Theisen, H., Vigil, S. A., \& Alaniz, V. M. (1993). Integrated solid waste management: engineering principles and management issues (Vol. 4). New York: McGraw-Hill.

[12] Pachauri, R. K., Allen, M. R., Barros, V. R., Broome, J., Cramer, W., Christ, R., ... \& Dubash, N. K. (2014). Climate change 2014: synthesis report. Contribution of Working Groups I, II and III to the fifth assessment report of the Intergovernmental Panel on Climate Change (p. 151). IPCC.

[13] Naciph, K., Rivadeneira, L., \& del Carmen Cazorla, M. (2013). Cálculo de las emisiones de C0 2 de la Universidad San Francisco de Quito pertenecientes al rubro de transporte estudiantil del Segundo Semestre 2012-2013. ACI Avances en Ciencias e Ingenierías, 5(2).

[14] Rivadeneira, L., Juncosa, J., Naciph, K., \& del Carmen Cazorla, M. (2014). Cálculo de las emisiones de C0 2 perteneciente al rubro de transporte del personal docente y administrativo de la Universidad San Francisco de Quito durante el Segundo Semestre 2013-2014. ACI Avances en Ciencias e Ingenierías, 6(1).

[15] ICA0.(2014). Carbon emissions calculator methodology - Versión 7. (p.16).

[16] Fisher, P.B. and E. McAdams. (2011). College of Charleston: 2011 Campus transportation study. Analysis of commuting habits and recommendations. College of Charleston, (p. 34). 